\title{
¿Cuándo seleccionar control del ritmo o control de la frecuencia en fibrilación auricular?
}

\section{When to select control of the rhythm or control of the frequency in atrial fibrillation?}

\author{
Víctor Manuel Velasco*, Fernando Rosas, Juan Felipe Betancourt, \\ Juan Manuel Camargo y David Santacruz
}

\author{
Departamento de Electrofisiología y Estimulación Cardiaca, Fundación Clínica Shaio, Bogotá, Colombia
}

Recibido el 3 de octubre de 2016; aceptado el 6 de octubre de 2016

Disponible en Internet el 9 de noviembre de 2016

La fibrilación auricular es un problema muy común y su prevalencia aumenta con la edad (35\% de los pacientes mayores de 80 años la tienen $)^{1}$ y con otros factores de riesgo (hipertensión, obesidad, diabetes, falla cardíaca, apnea del sueño, etc. $)^{2}$. Esta prevalencia alta se asocia además con un tratamiento costoso.

El tratamiento de la fibrilación auricular incluye los siguientes aspectos:

1. Manejo de la enfermedad subyacente y de los factores de riesgo para fibrilación auricular.

2. Prevención de tromboembolia.

3. Control de la frecuencia ventricular durante la fibrilación auricular (control de la frecuencia).

4. Restauración y mantenimiento del ritmo sinusal normal (control del ritmo).

En este artículo se discutirán los apartados 3 y 4: cuándo seleccionar control de la frecuencia o del ritmo en pacientes que no están en una fase aguda, descompensados. El manejo de urgencias en fibrilación se tratará en otros capítulos.

\footnotetext{
* Autor para correspondencia.

Correo electrónico: velascovm@gmail.com (V.M. Velasco).
}

\section{Control de la frecuencia}

El control de la frecuencia ventricular durante fibrilación auricular, es una consideración fundamental en todos los pacientes. Pocos casos no necesitan una terapia específica para el control de la frecuencia, y estos constituyen los pacientes que tienen enfermedad del sistema de conducción cardiaco. Este grupo requiere vigilancia dado que pueden evolucionar a bradicardia sintomática. Algunos casos tienen fibrilación auricular con frecuencia ventricular rápida, seguida de bradicardia durante ritmo sinusal por enfermedad del nodo sinusal asociada, lo cual se conoce como síndrome de bradicardia-taquicardia. Sin embargo, la bradicardia espontánea o inducida que se documenta al terminar un episodio de fibrilación auricular puede darse por disfunción transitoria del nodo sinusal. Si el control del ritmo de la fibrilación auricular no es posible, algunos pacientes pueden requerir implante de marcapasos definitivo para evitar la bradicardia y facilitar el manejo farmacológico ${ }^{3}$.

Los tres trabajos más extensos que analizan la estrategia de control de frecuencia versus el control del ritmo en fibrilación auricular, son los estudios AFFIRM, RACE y AF-CHF y todos llegan a la misma conclusión: el control del ritmo no es superior al control de la frecuencia en términos de morbilidad y mortalidad. La estrategia de control de la frecuencia es una opción legítima primaria de tratamiento. 
Según el AFFIRM ${ }^{4}$, que es el estudio más vasto, el objetivo del control de la frecuencia ventricular durante la fibrilación auricular, es lograr una frecuencia cardíaca en reposo menor o igual a 80 latidos por minuto. También se debe buscar una frecuencia cardiaca máxima durante la prueba de la caminata de los 6 minutos menor o igual a 110 latidos por minuto o durante la monitorización de Holter ambulatorio electrocardiográfico de 24 horas, una frecuencia cardiaca promedio menor o igual a 100 latidos por minuto. El control óptimo de la frecuencia es una estrategia importante; impacta en la calidad de vida, disminuye la morbilidad y reduce el riesgo de desarrollar miocardiopatía inducida por taquicardia.

Datos más recientes derivados del estudio RACE $^{5}$ en fibrilación auricular permanente, indican que una estrategia más laxa o indulgente de frecuencia (frecuencia cardíaca en reposo menor de 110 latidos por minuto), puede ser efectiva y fácil de lograr, y es una opción razonable en pacientes con función ventricular izquierda preservada.

El estudio $\mathrm{AF}_{-} \mathrm{CHF}^{6}$ se realizó en pacientes que tenían mala función ventricular y no mostró beneficios de control del ritmo versus control de la frecuencia en pacientes con fibrilación auricular y terapia médica óptima: anticoagulantes, betabloqueadores, inhibidores de la enzima convertidora de angiotensina ॥ y espironolactona. El estudio en mención confirmó que la estrategia de control de la frecuencia es un camino legítimo de tratamiento en los pacientes con falla cardiaca y resaltó la importancia de tratar las comorbilidades en los pacientes con fibrilación auricular.

Los agentes farmacológicos de mayor uso para lograr el control de la frecuencia son los betabloqueadores y los antagonistas de los canales de calcio no dihidropiridínicos (diltiazem o verapamilo). La digoxina se emplea comúnmente en combinación con los anteriores para obtener un control satisfactorio de la frecuencia, en tanto que la digoxina sola puede ser la terapia primaria en presencia de hipotensión o falla cardiaca ${ }^{7}$. En otros capítulos de estas guías se indica cómo se pueden seleccionar los medicamentos para el control de la frecuencia ventricular (con base en la severidad de los síntomas y de las comorbilidades) y en qué casos la ablación del nodo aurículo-ventricular y el implante de un dispositivo de estimulación cardíaca son una opción para pacientes con falla cardíaca importante y rápida respuesta ventricular después de una terapia farmacológica máxima o para pacientes con edad avanzada o pobre pronóstico que no son candidatos a una ablación.

\section{Recomendaciones}

Según las guías de 2014 de la AHA/ACC/HRS de manejo de la fibrilación auricular las recomendaciones para el control de frecuencia son las siguientes ${ }^{1}$ :

\section{Clase I}

- En fibrilación auricular paroxística, persistente o permanente se recomienda el control de la respuesta ventricular con betabloqueadores o antagonistas de los canales de calcio no dihidropiridínicos (nivel de evidencia B).

- Los pacientes que refieren síntomas durante una actividad física deben valorarse durante el ejercicio para realizar los ajustes farmacológicos necesarios (nivel de evidencia C).

\section{Clase ॥a}

- Una estrategia de control de frecuencia cardiaca (en reposo menor de 80 por minuto) es razonable en pacientes sintomáticos con fibrilación auricular (nivel de evidencia B).

- La ablación del nodo aurículo-ventricular con implante de marcapasos definitivo es una opción racional para el control de la frecuencia cardiaca cuando la terapia farmacológica es inadecuada y no se ha logrado estabilizar el ritmo (nivel evidencia $B$ ).

\section{Clase ॥b}

- Una estrategia del control de la frecuencia laxo o indulgente (frecuencia cardiaca en reposo menor de 110 latidos por minuto), puede ser útil a largo plazo en pacientes asintomáticos y con función ventricular izquierda preservada (nivel de evidencia B).

- Puede usarse amiodarona oral cuando otras medidas son ineficaces o están contraindicadas (nivel de evidencia C).

\section{Control del ritmo}

Las estrategias para restaurar y mantener el ritmo sinusal incluyen una combinación de procedimientos: cardioversión eléctrica o farmacológica, medicamentos antiarrítmicos, ablación con catéter o con criobalón y ablación quirúrgica. Algunos pacientes pueden necesitar marcapasos para prevenir bradicardia y pausas que faciliten la inducción de fibrilación auricular ${ }^{3}$.

Los medicamentos antiarrítmicos constituyen la primera línea de terapia para prevenir recurrencias de fibrilación auricular. Se utilizan bloqueadores de los canales de sodio (clase ।) como propafenona y flecainida o bloqueadores de los canales de potasio (clase III) como amiodarona y sotalol. Son medicamentos moderadamente efectivos. La recurrencia de fibrilación auricular con fármacos antiarrítmicos es de 50\% a 6-12 meses; además estas medicaciones tienen un perfil de seguridad limitado. Pueden prolongar el intervalo QT en el electrocardiograma y causar torsades de pointes y muerte súbita. Pueden convertir una fibrilación auricular en un flutter auricular con conducción 1:1 y respuesta ventricular muy rápida, de ahí que conviene asociarles bloqueadores del nodo aurículo-ventricular como los antagonistas de los canales de calcio no dihidropiridínicos o los betabloqueadores ${ }^{7}$.

Otra razón para considerar la terapia de control de ritmo con medicamentos antiarrítmicos como primera elección, es la de temporizar y de acuerdo con la evolución, determinar cuál es la mejor terapia a largo plazo, teniendo como objetivo final la ablación con catéter.

\section{Recomendaciones}

Según las guías de 2014 de la AHA/ACC/HRS las recomendaciones que se sugieren con los medicamentos antiarrítmicos para el mantenimiento de ritmo sinusal son las siguientes ${ }^{1}$ : 


\section{Clase I}

- Antes de iniciar medicamentos antiarrítmicos se deben controlar los factores precipitantes y las causas reversibles de fibrilación auricular (nivel de evidencia C).

- Se recomiendan los antiarrítmicos amiodarona, dofetilide, dronedarone, flecainida, propafenona y sotalol en fibrilación auricular para el mantenimiento del ritmo sinusal de acuerdo con la enfermedad cardíaca subyacente y las comorbilidades (nivel de evidencia A).

- Los riesgos de los antiarrítmicos, incluida la proarritmia, deben considerarse antes de iniciar terapia con cada medicamento (nivel de evidencia C).

- Por sus efectos tóxicos la amiodarona solo debe utilizarse después de analizar sus riesgos y cuando otros agentes hayan fallado o estén contraindicados (nivel de evidencia C).

\section{Clase ıa}

- La estrategia de control del ritmo con terapia farmacológica puede ser útil en los pacientes con fibrilación auricular para el tratamiento de la taquicardiomiopatía (nivel de evidencia C).

\section{Clase ॥b}

- Se puede continuar terapia con medicamentos antiarrítmicos cuando las recurrencias de fibrilación auricular son infrecuentes y bien toleradas y el fármaco ha reducido frecuencia o síntomas (nivel de evidencia C).

El reconocimiento realizado por Haissaguerre et al. en $1998^{8}$, de que la mayoría de los disparadores de fibrilación auricular se localizan en las venas pulmonares, cambió el manejo de la misma porque impulsó el desarrollo de la ablación para prevenir recurrencias de fibrilación auricular enfocada en el aislamiento de las venas pulmonares. Los mecanismos que mantienen o sostienen la fibrilación auricular no están claros y por esto las otras técnicas ablativas complementarias son empíricas: líneas de bloqueo en varias partes de las aurículas, ablación de los electrogramas fraccionados (CAFE) y ablación de los rotores y de los plexos gangliónicos ${ }^{7}$.

La ablación inicial en los casos de fibrilación auricular paroxística se realiza alrededor del antro de las venas pulmonares. La mayor eficacia se logra en los pacientes que tienen fibrilación auricular paroxística aislada (lone atrial fibrillation) y menos de 60 años de edad. Los pacientes con fibrilación auricular persistente requieren además ablación en otros sitios como la vena cava superior, el seno coronario y las paredes auriculares.

Varios factores influyen en la eficacia y seguridad de la ablación; estos son: el tipo de fibrilación auricular (paroxística versus persistente versus largamente persistente), el grado de síntomas y el número de comorbilidades. La mayoría de los estudios de ablación con catéter se han realizado en personas jóvenes y sanas con fibrilación auricular paroxística refractaria a una o más drogas antiarrítmicas. La seguridad y eficacia del procedimiento están menos establecidos en los pacientes con fibrilación auricular largamente persistente, en los muy ancianos y en aquellos con falla cardiaca significativa incluyendo taquicardiomiopatía ${ }^{1}$.
En el trabajo de Haissaguerre, utilizando como criterio de éxito en ablación de fibrilación auricular la ausencia de fibrilación auricular o de taquicardia auricular mayor de 30 segundos de duración, con una sola ablación, el procedimiento fue exitoso en el $40 \%, 37 \%$ y $29 \%$ a uno, dos y cinco años respectivamente. En los pacientes que requirieron dos o más ablaciones el porcentaje de éxito después del último procedimiento fue de 87,81 y $63 \%$ a uno, dos y cinco años, respectivamente ${ }^{7}$.

La reconexión de las venas pulmonares es la causa más frecuente de recurrencia de fibrilación auricular y la mayoría ocurre en los primeros seis meses postablación sin que indique falla del tratamiento a largo plazo. Se puede iniciar tratamiento con drogas antiarrítmicas que usualmente son más efectivas después de una ablación. En los casos con fibrilación auricular persistente postablación se debe practicar cardioversión. Algunos casos responden a los fármacos antiarrítmicos y en otros casos puede ser necesaria una segunda ablación.

Las complicaciones de la ablación de fibrilación auricular son: taponamiento cardiaco, accidentes cerebrovasculares, fístula atrioesofágica, estenosis de venas pulmonares, hematomas y pseudoaneurismas femorales, lesión de nervio frénico y taquicardias auriculares. La mortalidad relacionada con la ablación con catéter en fibrilación auricular es de 1 por 1.000. En un estudio mundial de seguimiento del procedimiento se encontraron complicaciones en el 4,5\% de los casos, pero si se incluye el flutter auricular atípico iatrogénico la tasa de complicaciones se eleva al $14 \%^{9}$.

El criobalón está disponible recientemente como un nuevo método para practicar ablación de fibrilación auricular. Proporciona una ablación circunferencial a diferencia de la ablación punto a punto que se realiza con radiofrecuencia. Los resultados agudos y a largo plazo son similares. Las complicaciones del procedimiento son muy parecidas excepto la incidencia de lesión del nervio frénico que con criobalón es más elevada (6\%); ocurre cuando se practica la ablación en la vena pulmonar superior derecha. La estrategia de estimular la región del nervio frénico cuando se realiza la ablación de las venas derechas vigilando los signos de parálisis del frénico, ha disminuido la incidencia de esta complicación ${ }^{10}$.

Aunque una estrategia inicial de control de la frecuencia es razonable para muchos pacientes, para otros existen consideraciones a favor del control del ritmo que deben analizarse. La restauración y el mantenimiento del ritmo sinusal se asocia con mejoría de los síntomas y de la calidad de vida. Otros factores a favor del control del ritmo incluyen la dificultad para lograr un control adecuado de la frecuencia, la edad en pacientes jóvenes, la taquicardiomiopatía, el primer episodio de fibrilación auricular y la preferencia del paciente. La progresión de fibrilación auricular de paroxística a permanente se asocia a remodelamiento eléctrico y estructural que puede ser irreversible. Una intervención precoz con estrategia de control del ritmo puede prevenir la progresión de la fibrilación auricular y ser benéfica ${ }^{3}$.

\section{Recomendación}

Las siguientes son las recomendaciones de las guías 2014 AHA/ACC/HRS acerca de las indicaciones de ablación con catéter para mantenimiento de ritmo sinusal ${ }^{1}$ : 


\section{Clase I}

- La ablación con catéter es útil en fibrilación auricular paroxística, sintomática, refractaria o con intolerancia al menos a una droga antiarrítmica clase I o clase III cuando la estrategia de control del ritmo es la deseada (nivel de evidencia A).

\section{Clase ॥a}

- La ablación con catéter es razonable en algunos pacientes con fibrilación auricular persistente, sintomática, refractaria o con intolerancia al menos a una medicación antiarrítmica clase । o clase III (nivel de evidencia A).

- En pacientes con fibrilación auricular paroxística, sintomática y recurrente, la ablación con catéter puede ser la estrategia inicial del control del ritmo antes de ensayos terapéuticos con medicamentos antiarrítmicos después de poner en la balanza los riesgos y beneficios de las drogas y de la ablación (nivel de evidencia B).

\section{Clase ॥b}

- La ablación con catéter puede considerarse en pacientes sintomáticos con fibrilación auricular largamente persistente (más de 12 meses), refractaria o con intolerancia al menos a una droga antiarrítmica clase I o clase II cuando la estrategia de control del ritmo es la deseada (nivel de evidencia B).

- La ablación con catéter puede considerarse antes de iniciar terapia antiarrítmica con drogas clase I 0 III en pacientes con fibrilación auricular persistente, sintomática cuando la estrategia de control del ritmo es la deseada (nivel de evidencia $B$ ).

En conclusión, ¿cuándo seleccionar control de la frecuencia o cuándo del ritmo? En algunos casos la elección de la estrategia es obvia y fácil. En otros, ambos caminos, control de la frecuencia o control del ritmo, son válidos. En esta situación se compromete al paciente y a su familia en el proceso de toma de decisiones. Se deben explicar los potenciales beneficios y riesgos de cada estrategia. Hay pacientes que comienzan con estrategia de control del ritmo y se enfrentan a una evolución tórpida, no satisfactoria, de ahí que pasan rápidamente a la estrategia de control de la frecuencia. En otros la historia se da al contrario; por tanto, se debe individualizar cada caso.

\section{Conflicto de intereses}

Los autores declaran no tener ningún conflicto de intereses.

\section{Bibliografía}

1. January CT, Wann LS, Alpert JS, et al. 2014AHA/ACC/HRS guideline for the management of patients with atrial fibrillation a report of the American College of Cardiology/American Heart Association Task Force on practice guidelines and the Heart Rhythm Society. Circulation. 2014;130: e199-267.

2. Go AS, Hyleck EM, Phillips KA, et al. Prevalence of diagnosed atrial fibrillation in adults: national implications for rhythm management and stroke prevention. The AnTicoagulation and Risk Factors in Atrial Fibrillation (ATRIA) Study. JAMA. 2001;285:2370-5.

3. Moukabary T, Gonzalez MD. Management of atrial fibrillation. Med Clin N Am. 2015;99:781-94.

4. Wyse DG, Waldo AL, DiMarco JP. A comparison of rate control and rhythm control in patients with atrial fibrillation. $\mathrm{N}$ Eng $\mathrm{J}$ Med. 2002;347:1825-33.

5. Van Gelder IC, Hagens VE, Bosker HA, et al. A comparison of rate control and rhythm control in patients with recurrent persistent atrial fibrillation. N Eng J Med. 2002;347:1834-40.

6. Roy D, Talajic M, Dorian P, et al. Rhytm control versus rate control for atrial fibrillation and heart failure. N Eng J Med. 2008;358:2667-77.

7. Waldo AL. Rate control versus rhythm control in atrial fibrillation: lessons learned from clinical trials of atrial fibrillation. Science Direct. 2015. Disponible en: www.sciencedirect.com.

8. Haissaguerre M, Jais P, Shah DC, et al. Spontaneous initiation of atrial fibrillation by ectopic beats originating in the pulmonary veins. N Eng J Med. 1998;339:659-66.

9. Cappato R, Calkins H, Chen SA, et al. Updated worldwide survey on the methods, efficacy and safety of catheter ablation for human atrial fibrillation. Circ Arrhythm Electrophysiol. 2010;3:32-8.

10. Andrade JG, Khairy P, Guerra PG, et al. Efficacy and safety of cryoballon ablation for atrial fibrillation: a systematic review of published studies. Heart Rhythm. 2011;8:1441-51. 